\title{
Designing mediation for context-aware applications
}

\author{
ANIND K. DEY \\ Intel Research, Berkeley \\ and \\ JENNIFER MANKOFF \\ EECS Dept., UC Berkeley
}

\begin{abstract}
Many context-aware services make the assumption that the context they use is completely accurate. However, in reality, both sensed and interpreted context is often ambiguous. A challenge facing the development of realistic and deployable context-aware services, therefore, is the ability to handle ambiguous context. Although some of this ambiguity may be resolved using automatic techniques, we argue that correct handling of ambiguous context will often need to involve the user. We use the term mediation to refer to the dialogue that ensues between the user and the system. In this paper, we describe an architecture that supports the building of context-aware services that assume context is ambiguous and allows for mediation of ambiguity by mobile users in aware environments. We discuss design guidelines that arise from supporting mediation over space and time, issues not present in the graphical user interface domain, where mediation has typically been used in the past. We illustrate the use of our architecture and the design guidelines and evaluate it through three example context-aware services, a word predictor system, an In/Out Board, and a reminder tool.
\end{abstract}

Categories and Subject Descriptors: H5.2 [Information Interfaces and Presentation]: User Interfaces Graphical user interfaces; Interaction styles; D.2.11 [Software Architectures]: Software Engineering Domain-specific architectures;

General Terms: Context-aware Computing, Ambiguity, Aware Environments, Ubiquitous computing, Mediation, Error handling

Additional Key Words and Phrases:

\section{INTRODUCTION}

A characteristic of an aware, sensor-rich environment is that it senses and reacts to context, information sensed about the environment's mobile occupants and their activities, by providing context-aware services that facilitate the occupants in their everyday actions. Researchers have been building tools and architectures to facilitate the creation of these context-aware services by providing ways to more easily acquire, represent and distribute raw sensed data and inferred data [30]. Our experience shows that though sensing is becoming more cost-effective and ubiquitous, the interpretation of sensed data as context is still imperfect and will likely remain so for some time. Yet historically this issue has often been ignored or glossed over. A challenge facing the development of realistic and deployable context-aware services, therefore, is the ability to handle imperfect, or ambiguous, context.

Authors' addresses: Anind K. Dey, Intel Research Berkeley, Intel Corporation, Berkeley, CA 94704; Jennifer Mankoff, EECS Department, UC-Berkeley, Berkeley, CA 94720-1770.

Permission to make digital/hard copy of part of this work for personal or classroom use is granted without fee provided that the copies are not made or distributed for profit or commercial advantage, the copyright notice, the title of the publication, and its date of appear, and notice is given that copying is by permission of the 
ACM, Inc. To copy otherwise, to republish, to post on servers, or to redistribute to lists, requires prior specific permission and/or a fee.

(C) 2001 ACM 1073-0516/01/0300-0034 \$5.00

Although some of this ambiguity may be resolved using automatic techniques, we argue that correct handling of ambiguous context will often need to involve the user. We and others have used the term mediation to refer to the dialogue that ensues between the user and the system $[28,21,36]$. This can be seen as an application of mixed-initiative interaction to the problem of correcting ambiguity [19,21,23,27], and was originally inspired by the concept of grounding, the signals that humans use during conversation to disambiguate potential misunderstandings [9]. Mediation techniques are interface elements that help the user to identify and fix system actions that are incorrect, or potentially involve the user in helping the system to avoid making those mistakes in the first place. This touches on two key challenges for designing the communication aspects of sensing-based systems, highlighted recently by Bellotti et al. [3]. Bellotti et al identify five challenges in all: how to address individual devices in a sensor-rich environment, how to know that the system is attending to the user, how to take action, how to know that the system has taken the correct action, and how to avoid mistakes. Mediation addresses the issues of providing feedback to support users in knowing that the system is attending to them and in knowing what the system has done and providing the ability to disambiguate sensed input to avoid the system taking incorrect actions.

This paper includes two contributions relating to ambiguity management: First, we present design guidelines for mediation in sensing-based systems that address design issues beyond those normally dealt with in graphical user interfaces (GUIs). We illustrate these guidelines using three typical context-aware applications. Second, we discuss our architectural support for mediation, feedback and disambiguation. We have built a runtime architecture that supports programmers in the development of multi-user, interactive, distributed applications that use ambiguous data.

\subsection{Designing for Ambiguity}

During the course of our research in sensor-based interactions, we have built a number of applications that use ambiguous context and support users in mediating this ambiguity. Ambiguous context, from an aware environment, can produce errors similar to those in desktop recognition-based interfaces. Just as a speech recognizer can incorrectly recognize a user's utterances, a positioning system using Wi-Fi signal strength can incorrectly locate a user. In both cases, a user's actions may be misinterpreted, or missed entirely. Additionally, lack of input may be mistaken as an action. Ambiguity arises when a recognizer is uncertain as to the current interpretation of the user's input, as defined by the user's intent. An application can choose to ignore the ambiguity and just take some action (e.g. act on the most likely choice), or can use mediation techniques to ask the 
user about her actual intent. In our past work in desktop interface design, we used mediation to refer to the dialogue between the user and computer that resolves questions about how the user's input should be interpreted in the presence of ambiguity [28]. A common example of mediation in recognition-based desktop interfaces is the $n$-best list, where the $n$ most likely interpretations of some ambiguous input are presented to the user to choose from. Mediation can be seen as an application of mixed initiative interaction [23], or humans and computers solving problems together, to the problem of ambiguity and error correction.

While a known set of mediation techniques can be applied in most instances of desktop-based interfaces [28], in the case of ambiguous context, there are additional challenges that arise for several reasons. In particular, humans in off-the-desktop environments are mobile and may be involved in much more complex situations than in desktop environments. Additionally, input is often implicit, and a person may not be interacting with a computer at all when ambiguity needs to be resolved. These and other issues led us to develop the following guidelines for mediation of context-aware applications:

- Applications should provide redundant mediation techniques to support more natural and smooth interactions;

- Applications should facilitate providing input and output that are distributed both in space and time to support input and feedback for mobile users;

- Interpretations of ambiguous context should have carefully chosen defaults to minimize user mediation, particularly when users are not directly interacting with a system.

- Ambiguity should be retained until mediation is necessary for an application to proceed.

A valid question is: Why involve users in mediation at all? Certainly, mediation puts a burden on the user by asking him to help the system out. Why not simply improve recognition, extend the number of sensors in use, and save the user the effort? Our answer is that currently, the information available to recognizers is simply too limited to support the level of certainty necessary to eliminate the need for user feedback. Indeed, the ultimate sensing-based recognition system, the human being herself, often uses dialogue to resolve uncertainty when conversing with other humans.

\subsection{System Architecture}

Designing correction strategies that meet these requirements can be facilitated by architectural support. In previous work, we presented an architecture for the development of context-aware services that assumed context to be unambiguous [14]. We also 
developed an architecture to support the mediation of ambiguity in recognition-based GUI interfaces [28]. Building on this past work, we developed support for the additional architectural requirements that arise as a result of requesting highly mobile users to mediate ambiguous context in distributed, interactive, sensing environments [13]. Our architecture supports the building of applications that allow humans in an aware environment to detect errors in sensed information about them and their intentions and correct those errors in a variety of ways. In particular it supports:

- Acquisition of ambiguous context;

- Context mediation;

- Delivery of ambiguous context to multiple applications that may or may not be able to support mediation;

- Pre-emption of mediation by another application or component;

- Applications or services in requesting that another application or service mediate;

- Distributed feedback about ambiguity to users in an aware environment; and,

- Delayed storage of context once ambiguity is resolved.

Our runtime architecture addresses these issues and supports our goal of building more realistic context-aware applications that can handle ambiguous data through mediation.

\subsection{Overview of Paper}

We begin by presenting related work. In the next section, we present a motivating example used to illustrate the requirements of mediation in a context-aware setting, followed by a discussion of mediation from an application designer's perspective, and design guidelines for mediation. Next, we present requirements for our architecture, followed by brief overviews of previous work that we have extended: the Context Toolkit, an infrastructure to support the rapid development of context-aware services, which has assumed perfect context sensing in the past; and OOPS (Organized Option Pruning System), an architecture for the mediation of errors in recognition-based interfaces. We show how they were combined to deal with ambiguous context, and describe additional architectural mechanisms that were developed for the requirements unique to mediation of context in a distributed setting. We then present three case studies that illustrate how the architecture supports mediation of ambiguous context and how the design guidelines can be applied. The three applications are a word prediction system for the disabled (our motivating example), an In/Out Board and a context-aware reminder 
system. We conclude the paper with a discussion of further challenges in mediating interactions in context-aware applications.

\section{RELATED WORK}

Over the past several years, there have been a number of research efforts aimed at creating a ubiquitous computing environment, as described by Weiser [39]. In this section, we first define mediation, and then focus on those efforts dealing with context-aware systems and ambiguity. In particular, we divide these efforts into three categories: Context-aware applications; architectures to support context-aware services; and guidelines for dealing with ambiguity.

\subsection{Defining Mediation}

We define mediation as a dialog between a human and computer that resolves ambiguity. Mediation can conceptually be applied whenever misunderstandings arise between application and user (or even as a way of avoiding such misunderstandings), and it has played an important role in interface design since the earliest computer systems. We are particularly interested in the forms such a dialog can take (such as repeating input, selecting a choice from a list, and so on); and the architectural support needed to support mediation.

Particularly difficult problems arise when human input is misinterpreted by an application, an increasingly common phenomenon in interfaces, and one that poses serious usability and adoptability issues for users. Thus, our work is focused particularly on the design of and architectural support for mediators for resolving ambiguity caused by flaws in an application's understanding of its user.

\subsection{Current support for sensing and ambiguity in context-aware applications}

A system is context-aware if it uses context to provide relevant information and/or services to the user, where relevancy depends on the user's task. Context refers to information that can be used to characterize the situation of a person, place, or object relevant to the task at hand. Context may include information about activity, identity, location, and time. A classic example of a context-aware application is a tour guide that provides relevant information and/or services to a user based on her location $[2,7,8]$.

Table 1 presents a collection of existing context-aware applications, a short description of each system, the number of types of context a system senses, and how each system handles ambiguity. As shown in Table 1, a typical context-aware application includes only a small variety of sensed context (average is 2.35 and median is 2) [16]. An exception to this is Horvitz's Notification Platform [21], which uses seven separate sources of context. More typical is a tour guide, such as Cyberguide [2], which uses a 
location sensor and the identity of the current user (sensed statically via user login) as context.

Table 1: Uses of context and support for ambiguity in representative systems.

\begin{tabular}{|c|c|c|c|}
\hline System Name & System Description & $\begin{array}{l}\text { Number of Types } \\
\text { of Context }\end{array}$ & $\begin{array}{l}\text { Handling of } \\
\text { Ambiguity }\end{array}$ \\
\hline Classroom $2000[1]$ & Capture of a classroom lecture & 1 & Ignored \\
\hline GUIDE [8] & Tour guide & 1 & Ignored \\
\hline NETMAN [25] & Network maintenance & 1 & Ignored \\
\hline Active Badge [38] & Call forwarding & 1 & Ignored \\
\hline Fieldwork [33] & Fieldwork data collection & 1 & Ignored \\
\hline Stick-e Documents $[6,7]$ & Tour guide & 1 & Ignored \\
\hline Context Toolkit [14] & In/out board & 1 & Ignored \\
\hline Context Toolkit [14] & $\begin{array}{l}\text { Capture of serendipitous } \\
\text { meetings }\end{array}$ & 2 & Ignored \\
\hline Stick-e Documents $[6,7]$ & Paging and reminders & 2 & Ignored \\
\hline Augment-able Reality [34] & Virtual post-it notes & 2 & Ignored \\
\hline Cyberguide [2] & Tour guide & 2 & Ignored \\
\hline Teleport [5] & Migrating desktop environment & 2 & Ignored \\
\hline Responsive Office [18] & Office environment control & 4 & Ignored \\
\hline Reactive Room [11] & Intelligent control of audiovisuals & 6 & Ignored \\
\hline CyberDesk [17] & $\begin{array}{l}\text { Automatic integration of user } \\
\text { services }\end{array}$ & 6 & Ignored \\
\hline KidsRoom [4] & Interactive narrative space & 2 & $\begin{array}{l}\text { Automatic } \\
\text { Mediation }\end{array}$ \\
\hline Remembrance Agent [35] & $\begin{array}{l}\text { Suggests documents related to } \\
\text { active typing }\end{array}$ & 1 & Mediation \\
\hline QuickSet [10] & Interactive maps & 2 & Mediation \\
\hline LookOut [23] & $\begin{array}{l}\text { An email-based appointment } \\
\text { scheduling system }\end{array}$ & 2 & $\begin{array}{l}\text { Mixed-initiative, } \\
\text { Mediation }\end{array}$ \\
\hline Notification Platform [21] & A notification system & 7 & Mixed-initiative \\
\hline
\end{tabular}

The sensing of context is typically implicit where sensors are used to gather information without requiring action by the user. The more implicit the sensing, the more likely the chance there will be an error in its interpretation. However most of the applications featured in Table 1 ignore any uncertainty in the sensed data and its interpretations (these are labeled "Ignored" in the final column). In these applications, if the environment takes an action on incorrectly sensed input, it is the occupant's responsibility to undo the incorrect action (if this is possible) and to try again. There is no explicit support for users to handle or correct uncertainty in the sensed data and its interpretations.

However, some exceptions exist. The KidsRoom project uses computer vision to determine the location and activity of children in an interactive storytelling environment [4]. It attempts to constrain user interactions and uses automatic mediation techniques to resolve ambiguity when necessary. Automatic mediation is used to handle the uncertainty in the computer vision system, where an activity is selected when the probability of that activity crosses a pre-specified threshold. While automatic mediation is effective in many settings, in situations that are highly uncertain, it may not accurately be able to interpret 
user input. Thus, it is often the case that a dialog with the user is required to remove uncertainty. We now introduce a number of systems that use mediation to handle ambiguity in user input.

The Remembrance Agent selects relevant documents based on a user's typing activity, displays an $n$-best list of top documents (a standard form of mediation used in desktop applications) [35]. QuickSet, a multi-modal map application prompts the user for more information where uncertainty exists [10]. These applications all include mediation of some form, and we label them "Mediation" in Table 1. Work in the AI community on "mixed-initiative" interaction, which most broadly refers to humans and computers solving problems together, has often focused on handling of ambiguity or uncertainty $[19,21,23,27]$. For example, the LookOut system watches a user's email for potential appointments [23]. If it is fairly certain of an appointment, it will take the initiative to complete as much of the scheduling task as possible for a user. Alternatively, the user may take the initiative, invoking LookOut when the system chooses not to act due to uncertainty. LookOut also includes intermediate level actions such as simply confirming a potential appointment with the user. Before selecting an action, the system attempts to predict when it is appropriate to engage the user in dialog based on a measure of user attention. In no case does LookOut completely schedule an appointment without some sort of user confirmation. Mixed-initiative computing has also been applied in the context-aware computing domain. Examples include the Bayesian Receptionist, a speechbased, software receptionist [27], and the Notification Platform [21], a context-aware system that acts as a clearinghouse for incoming messages, dispatching them to a variety of devices based on context about a user's activity level and business.

\subsection{Systematic architectural support for context and ambiguity}

A number of architectures that facilitate the building of context-aware services, such as those shown in Table 1, have been built [6,12,14,20,24,37]. Unfortunately, as in the case of most context-aware applications, a simplifying assumption is made in all of these architectures, that the context being implicitly sensed is $100 \%$ certain. Context-aware services that are built on top of these architectures act on the provided context without any knowledge that the context is potentially uncertain. Our goal is to provide a general, reusable architecture that supports ambiguity and a variety of mediation techniques, ranging from implicit to explicit, that can be applied to context-aware applications. By removing the simplifying assumption that all context is certain, we are attempting to facilitate the building of more realistic services.

\subsection{Guidelines for dealing with ambiguity}


Although ambiguity is rarely addressed in context-aware systems, it has received some attention. In particular, Bellotti et al. raise multiple challenges relating to whether a user can find out if the system has done what they intended, find out about mistakes that have occurred, and correct mistakes in a timely manner [3]. They suggest two main solutions, relating to feedback and control. In terms of feedback, they advocate the following: Make sure that users can tell or ask what state a system is in; make feedback both timely and appropriate; make sure that feedback about the state, versus action or response, are easily differentiable. In terms of control, they advocate the following, both "in time" to avoid crucial system errors: Make sure that users can cancel or undo an action; help users disambiguate. Both feedback and control are the heart of what mediation is about, and the goal of our work is both to provide better, systematic support for mediation, and to suggest guidelines for effective mediation.

Prior to Bellotti et al.'s work, Horvitz proposed guidelines for mixed-initiative interaction, many of which are applicable to the problem of dealing with uncertainty or ambiguity. As he suggests in principle (5) one may "[employ] dialog to resolve key uncertainties," and (9) "[provide] mechanisms for efficient agent-user collaboration to refine results." This is what mediators are intended to do. He also makes some suggestions about how the impact of uncertainty on the user should be minimized, including (7) "minimizing the cost of poor guesses... including appropriate timing out..." and (8) "giving agents the ability to gracefully degrade" and "scoping precision of service to mask uncertainty." While these principles for mixed-initiative computing hold true for ambiguous, context-aware applications, there are additional guidelines that specifically relate to systems with mobile users and implicit input that we have discovered based on our work in this area. We present our guidelines in Section 4.

\subsection{Summary of related work}

It is the canonical, and very common, context-aware application, which includes two pieces of sensed data, simple intelligence if any, and (currently) no concept of ambiguity, that we are hoping to better support with our approach. By empowering the developers of such applications to consider and deal with ambiguity appropriately, we believe we can improve the experience of users of these systems. We believe that this can only happen when systematic, architectural support exists for handling ambiguity and experimenting with mediation strategies in context-aware applications.

\section{MOTIVATING EXAMPLE}

We have developed three applications as demonstrations of our architecture. One in particular, a context-aware communication system, the Communicator, will be used to illustrate key points throughout this paper, and we introduce it here. 
The Communicator is designed for people with motor and speech impairments. For these people, exemplified by Stephen Hawking, computers can provide a way to communicate with the world and increase both independence and freedom. Many people with severe motor impairments can control only a single switch, triggered by a muscle that is less spastic or paralyzed than others. This switch is used to scan through screen elements, such as the keys of a soft keyboard. Input of this sort is very slow and is often enhanced by word prediction. Our system is intended to support communication for a mobile user, both with co-located communication partners, and with remote communication partners. Ideally it will give its user the freedom to communicate currently enjoyed by a person who can speak to other nearby people, or call a friend with a cell-phone.
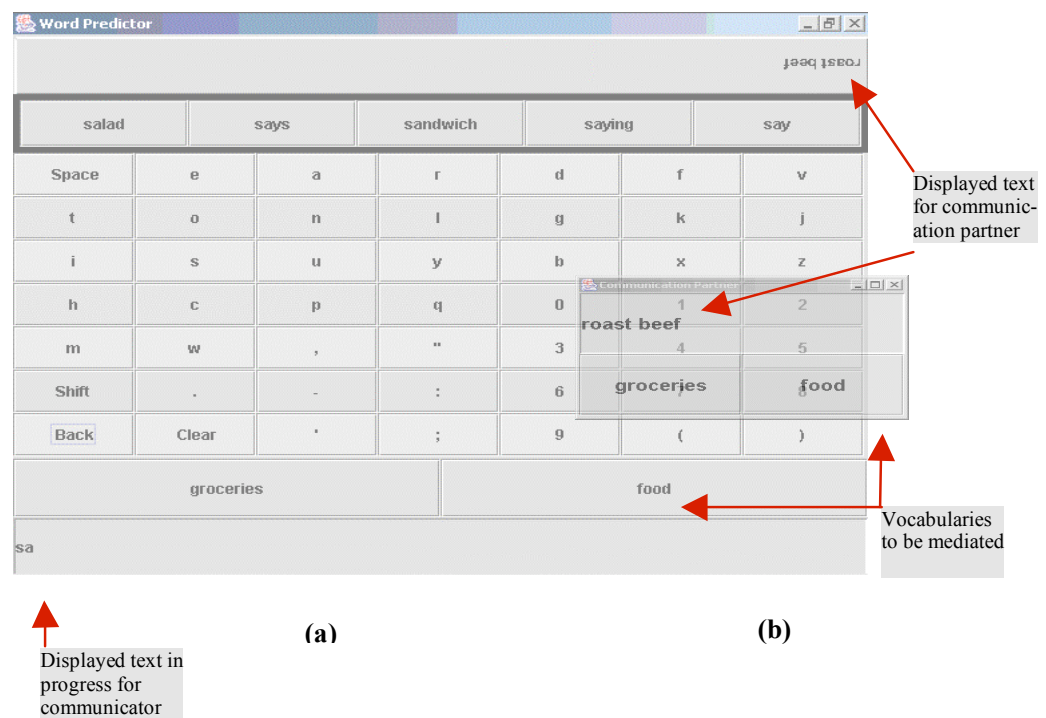

(a)

(b)

Figure 1 (a) Communicator (back) and (b) partner (right, front) interfaces.

The Communicator, shown in Figure 1, is based on a word predictor that attempts to predict what word a user is typing from the letters that have been typed so far. The nonspeaking individual uses the interface shown in Figure 1a. The keyboard layout shown was chosen for optimal efficiency for scanning input [26]. Text is displayed to the (abled) communication partner either at the top, reversed for easy readability by someone facing the user, across a flat display (Figure 1a, top); or on the display of a remote computer (Figure 1b). Word predictors are very inaccurate, and because of this, they usually display a list of possible predictions that the user scans through for the correct choice, often to no avail. Word prediction is especially difficult to use for spoken communication because the 
speed of conversational speech often reaches 120 words per minute (wpm) or more, while users of word prediction rarely go above $10 \mathrm{wpm}$.

The goal of the Communicator is to facilitate conversational speech through improved word prediction. We augment word prediction by using a third party intelligent system, the Remembrance Agent [35], to select conversation topics, or vocabularies, based on contextual information including recent words used, a history of the user's previous conversations tagged with location and time information, the current time and date and the user's current location. These vocabularies help to limit the set of predicted words to those that are more relevant and thus improve prediction. For example, when in a bank, words such as "finance" and "money" should be given priority over other similar words. This has been shown to be effective for predicting URLs in Netscape ${ }^{\mathrm{TM}}$ and Internet Explorer $^{\mathrm{TM}}$ and, in theory, for non-speaking individuals [26,29]. Our goal was to build an application to support context-aware word prediction for non-speaking individuals.

Unfortunately, it is hard to accurately predict the topic of a user's conversation, and because of this, the vocabulary selection process is ambiguous. We experimented with several mediation strategies, ranging from simply and automatically selecting the top choice vocabulary without user intervention to stopping the conversation to ask the user, or asking the communication partner, which vocabulary is correct.

We choose to use the Communicator as a running example because it is representative of the context-aware applications (discussed in the previous section) that we want to support. It includes three types of context, including sensed context (location), "sensor-fusion" (word prediction combines context about the optimal vocabulary with keyboard input) and "soft" context (conversational history, recent words), is mobile, distributed, and involves multiple users using different applications to view the same data stream. Additionally, it involves two ambiguous data sources: Location (where ambiguity is inherent in the sensor), and associated vocabularies, and word prediction (where ambiguity is inherent in the interpretation of sensed input).

\section{APPLICATION DESIGN CONSIDERATIONS: EXPLORING DISTRIBUTED MEDIATION IN PRACTICE}

This section presents a practical description of the key actions a programmer must take in developing a mediation-enriched application, including design guidelines for mediating sensor-based applications. Our past work with the Context Toolkit led to an architecture that supported the following design process for building a context-aware application [14]:

1. Specification. Specify the problem being addressed at a high level, including context-aware behaviors, and necessary context. 
2. Acquisition. Determine what new hardware and sensors are necessary to provide that context, write any necessary software and so on. (only necessary if sensors are not already supported)

3. Action. If appropriate, activate software to perform context-aware behavior.

In the Context Toolkit, step 3, action, is left entirely in the hands of the application designer, and is not supported by any toolkit mechanisms. Action is essentially what happens after context is handed off to an application, or any intermediary component that will act on context. Such an application or component may or may not directly involve a user in its action. It might explicitly involve the user, as in the case of the Communicator. It might change the environment, implicitly conveying information to the user by displaying something visually or in audio. Or it might simply update its state, or that of the environment, without any intent of notifying the user (for example, by modifying the climate control mechanisms in the environment).

But how should the design process change if the context that an application receives is ambiguous? Specification should remain unchanged except for the choice to use ambiguous context. Acquisition also remains the same, with the sole requirement that the system not throw away ambiguity, but instead pass it to the application to mediate. Only action is really affected. In particular, we believe that an application designer should include a plan for dealing with ambiguity in her application design, and that the elements of this plan can be conceptualized as mediators, and can be systematically supported by the toolkit. This approach is based in our past work in mediation of Graphical User Interfaces [28]. From a practical perspective, this means that the application designer should separate her handling of ambiguity from the rest of the application interface. A mediator, then, is a component that represents one particular way of handling ambiguity in one or more context sources. In the Communicator, the row of buttons labeled "Vocabularies to be mediated" is a mediator. A mediator represents a dialog. This is appropriate in a setting where user involvement is explicit. When the connection to a user is implicit, a mediator may simply display information at a different level of precision appropriate to the amount of ambiguity present. If no user is present, a mediator may choose to pause action until uncertainty is resolved, try to contact the user, or do it's best to automatically determine the top choice.

Based on our work in developing three ambiguous, context-aware applications, we present the following practical guidelines for designing mediation into context-aware applications.

- Applications should provide redundant mediation techniques to support more natural and smooth interactions; 
- Applications should provide facilities for providing input and output that are distributed both in space and time to support input and feedback for mobile users;

- Interpretations of ambiguous context should have carefully chosen defaults to minimize user mediation, particularly when users are not directly interacting with a system.

- Ambiguity should be retained until mediation is necessary for an application to proceed.

Below, we present explanations of each guideline. Note that while these may seem obvious in retrospect, our contribution lies not only in identifying them, but also in illustrating how the unique needs of mobile users and context-aware applications require that these issues be addressed.

\subsection{Providing Redundant Mediation Techniques}

One of the attractive features of context-aware computing is the promise that it will allow humans to carry out their everyday tasks without having to provide additional explicit cues to some computational service. Our experience shows, however, that the more implicit the gathering of context, the more likely it is to be in error. In the GUI domain, typically only two mediation techniques are provided at a time, an $n$-best list of some sort, and a way to delete or undo incorrect interpretations and re-enter them. In designing mediation techniques for correcting context, a variety of redundant techniques should be provided simultaneously. This redundant set not only provides a choice on the form of user input and system feedback, but also the relative positioning and accessibility to the user should be carefully thought out to provide a smooth transition from most implicit (and presumably least obtrusive) to most explicit [35]. This gives the user the freedom to select the most appropriate level of interaction, based on the seriousness of any errors, and her own level of engagement in the task. This is similar to the set of social interactions that may occur when someone knocks on one's office door. If you are on the phone, you might talk louder (or more quietly), and ignore the knock. If you are not at all busy, you might walk to the door to answer it. A range of intermediate responses exists as well. Additionally, because recognition is less accurate in unconstrained, mobile settings, it is particularly crucial to provide redundancy. If one mediation technique fails due to recognition errors, other options are still available.

\subsection{Spatio-temporal Relationship of Input and Output}

Some input must be sensed before any interpretation and subsequent mediation can occur. Because we are assuming user mobility, this means that the spatial relationship of initial input sensors must mesh with the temporal constraints to interpret that sensed input 
before providing initial feedback to the user. Should the user determine that some mediation is necessary, that feedback needs to be located within physical range of the sensing technologies used to mediate the context and the space through which the user is moving. In contrast, both the user's attention and location are relatively fixed in the GUI domain. Mediating context should occur along the natural path that the user would take. In some cases, this might require duplicate sensing technologies to take into account different initial directions in which a user may be walking. In addition, the mediation techniques may need to have a carefully calculated timeout period, after which mediation is assumed not to happen, because a user may not have noticed or may have moved past a mediator.

\subsection{Effective Use of Defaults}

Sometimes the most effective and pleasurable interactions are ones that do not have to happen. Prudent choices of default interpretations can result in no additional correction being required from the user. These defaults could either provide some default action or provide no action, based on the situation. For example, in a situation involving highly ambiguous context such as word prediction, it may be best to do nothing by default and only take action if the user indicates the correct interpretation through mediation. This

guideline is important in the GUI domain, but crucial in the context domain because the amount of ambiguous context is likely to be more than a user could be reasonably expected to handle.

\subsection{Ambiguity should be retained}

If every ambiguously sensed event was mediated, a user could be overwhelmed with requests for confirmation. However, ambiguity may be retained in two circumstances. First, there is no reason to ask a user for input until an application needs to act on the sensed data. Second, if the application can use the data even while ambiguous, it should. An example is the use of vocabularies in the communicator. Vocabularies for the top choices can all be merged, in the absence of user feedback about which is correct. Again, these issues exist in the GUI domain, but are more crucial in the context domain because it can reduce the number and frequency of mediation requests the user is faced with.

\section{ARCHITECTURE REQUIREMENTS}

The focus of this paper is on support for building context-aware applications that deal realistically with ambiguity. This includes providing design guidelines for building applications and addressing the architectural issues needed to deliver ambiguous context and support its mediation. On the architecture side, there must exist a system that is able to capture context and deliver it to interested consumers, and there must be support for 
managing ambiguity. Our architecture addresses seven challenges that arise from mediating ambiguous context.

1) Context Acquisition and Ambiguity: One common characteristic of context-aware applications is the use of sensors to collect data. In the Communicator, location and time information is used to help improve word prediction. A user's location could be sensed using Active Badges, radar, video cameras or GPS units. All of these sensors have some degree of ambiguity in the data they sense. A vision system that is targeted to identify and locate users based on the color of the clothing they wear will produce inaccurate results if multiple users are wearing the same color of clothing. The ambiguity problem is made worse when applications derive implicit higher-level context from sensor data. This issue arises in the Communicator's inference about vocabulary from (already ambiguous) location and time. Even with the use of sophisticated AI techniques, low- and high-level inferences are not always correct, resulting in ambiguity.

2) Context Mediation: As argued above, mediation is one way of resolving ambiguity. Architecturally, this leads to the following requirements: The architecture must have a model of ambiguity in sensed and interpreted data; it must be able to identify ambiguity when it is present, it must provide support for selecting among potential mediators and automatically instantiating them when ambiguous data arrives in an application. Additionally, an application developer must have some way to specify the relationship between data and mediator selection. Finally, a basic mediator class must be provided that includes simple functionality such as selecting a specific event as correct. In the GUI realm, we found that architectural support for these activities significantly reduces the amount of custom code a developer must write for each mediator [28].

3) Multiple Subscription Options: In many context-aware systems, multiple subscribers are interested in a single piece of sensed input. An interesting issue is how to allow individual components to "opt in" to ambiguous context while allowing others to "opt out". Some components may wish to deal with ambiguity while others may not. For example, non-interactive components such as a data logging system may not have any way to interact with users and therefore may not support mediation. The ability to opt out also makes our toolkit compatible with applications that cannot handle ambiguous context such as previously existing Context Toolkit applications.

Other components may only wish to receive unambiguous data. For example, a logging system might wish to only record data that is certain. A second issue to deal with is allowing components to deal with ambiguous data while not requiring them to 
perform mediation. Later in the paper, we will discuss a word predictor widget in the Communicator that has this property.

4) Pre-emption of Mediation: In our system, multiple, completely unrelated components may subscribe to the same ambiguous data source. Both Communicator interfaces have the ability to mediate ambiguous vocabularies, for example. What should happen if one person selects one vocabulary while another selects a different one? We believe that multiple, conflicting disambiguations should not be allowed to exist simultaneously. Rather, disambiguation is meant to represent a human decision about the correct interpretation for ambiguous data. Where conflicts exist due to the presence of multiple users, we argue that it becomes a social issue, and should be dealt with as such. Note that we are not advocating multiple competing mediators within a single application, but rather that we need a way to handle conflicts when they occur across applications.

5) Forced Mediation: There are cases where a subscriber does not wish to mediate ambiguous data itself, but may still wish to exert some control over the timing of when another subscriber completes mediation. One way of doing this is allowing it to request immediate mediation by others. In the Communicator, when a conversation ends, a component responsible for managing past conversations wants to store this conversation in an appropriate vocabulary. This component does not have an interface, so it requests that the application mediate the possible vocabularies.

6) Feedback: When distributed sensors collect context about a user, a context-aware system needs to be able to provide feedback about the ambiguous context to her, particularly when the consequences are important to her, even where no interactive application is present. For this reason, the architecture needs to support the use of remote feedback, providing feedback (visual or aural, in practice) on any nearby device. For example, in a scenario where a user is being tracked by video, a device on the wall may display a window or use synthesized speech to indicate who the video camera system thinks the user is. This device is neither a subscriber of the context nor the context sensor, but simply has the ability to provide useful feedback to users about the state of the system. Note that we are not advocating constant feedback about all sensed events, but simply architectural support for the ability to provide feedback when something the user would want to know about is occurring, as determined by the application developer.

7) Storage: Because context-aware systems are often distributed and asynchronous, and because sensor data may be used by multiple applications, it is beneficial to store data being gathered by sensors. The Communicator takes advantage of stored information 
by accessing past conversations that match the user's current location and time. Storing context data allows applications that were not running at the time the data was collected to access and use this historical data. When that data is ambiguous, several versions must be saved, making the storage requirements prohibitive. Interesting issues to address are when should we store data (before or after ambiguity is resolved) and what should we store (ambiguous or unambiguous context).

In the next section, we will discuss the architecture we designed and implemented to deal with these requirements.

\section{MEDIATING AMBIGUOUS CONTEXT}

We built support for mediation of imperfectly sensed context by extending an existing toolkit, the Context Toolkit [14]. The Context Toolkit is a software toolkit for building context-aware services that support mobile users in aware environments, using context it assumes to be perfect. The toolkit makes it easy to add the use of context or implicit input to existing applications that do not use context. There are two basic building blocks that are relevant to this discussion: context widgets and context interpreters. Figure 2 shows the relationship between context components and applications.

Widgets and interpreters are intended to be persistent, running 24 hours a day, 7 days a week. They are instantiated and executed independently of each other in separate threads and on separate computing devices. The Context Toolkit makes the distribution of the context architecture transparent to context-aware applications, handling all communications between applications and components.

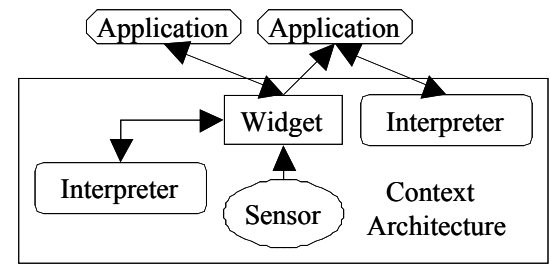

Figure 2 Context Toolkit components: arrows indicate data flow.

Context widgets are based on an analogy to GUI widgets. They encapsulate information about a single piece of context, such as location or activity, and provide a uniform interface to components that use the context. This makes it possible to use heterogeneous sensors to sense redundant input. Widgets maintain a persistent record of the context they sense. They allow applications and other widgets to both query and subscribe to the context information they maintain. The existing toolkit includes an extensible library of such widgets for sensing motion, temperature, identity (iButtons), volume level, and many others. Applications may use any combination of these sensors.

A context interpreter is used to abstract or interpret context. For example, if a GPS widget provides location context in the form of latitude and longitude, a context 
interpreter would be used to translate this to a street name. A more complex interpreter may take context from many widgets in a conference room to infer that a meeting is taking place. Both interpreters and widgets are sources of ambiguous data. A sensor may have inherent inaccuracies which affect the way a widget encapsulates its data, while an interpreter, because it is making inferences, naturally may include uncertainty.

\subsection{Modifications for Mediation}

In order to explain how we met the requirements given in the previous section, we must first introduce the basic abstractions we use to support mediation. We chose to base our work on the abstractions first presented in the OOPS toolkit [28], a GUI toolkit that provides support for building interfaces that make use of recognizers (e.g. speech, gestures) that interpret user input. Like sensing context, recognition is ambiguous and OOPS provides support for tracking and mediating uncertainty. We chose OOPS because it explicitly supports mediation of single-user, single application, non-distributed, ambiguous desktop input, a restricted version of our problem.

OOPS provides an internal model of recognized input, based on the concept of hierarchical events [32], which allows separation of mediation from recognition and from the application. This is a key abstraction that we will use in the extended Context Toolkit. This model encapsulates information about ambiguity and the relationships between input and interpretations of that input that are produced by recognizers in a graph (See Figure 3). The graph depicts relationships between source events, and their interpretations (which are produced by one or more recognizers). Note that this graph is not intended to encode semantic relationships or to support reasoning about those relationships. Thus the authoring of the graph is a simple matter of recording the source events from which interpretations are derived, and no explicit design is required to create the graph. The graph is used simply to identify the presence of ambiguity, and to ensure that the appropriate components are updated when decisions about ambiguity are made.

In most existing context-aware applications, the relationships represented in the graph are typically discarded along with any information about ambiguity. However, the creation of the graph is very straightforward since context events are being created each time an event is interpreted: when a new interpretation is created in a widget or interpreter, it must be passed a set containing any source events that were used to create it. This is done at creation time, when that information is still easily accessible, and simply requires an additional argument to the event constructor. More importantly, when ambiguity is present, multiple events must be created for each ambiguous interpretation. This postpones the work of selecting the most likely choice for later, and moves that work to the mediation phase of event handling. 


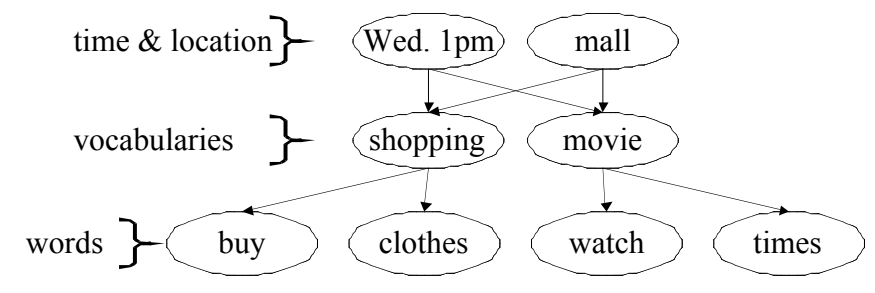

Figure 3 An event graph representing predicted words from context.

Like OOPS, our toolkit automatically identifies ambiguity in the graph and intervenes between widgets and interpreters and the application by passing the directed graph to a mediator. A mediator is a component in the application that either resolves ambiguity automatically, or allows the user and computer to communicate about ambiguity. Mediators generally fall into three major categories. Choice mediators give the user a choice of possible interpretations of her input. Repetition mediators support the user in repeating her input, usually in an alternate and less error-prone modality. Both types of mediators are very similar to a GUI widget such as a button or menu, with the exception that they only appear when ambiguity that they are designed to resolve is present. Automatic mediators select the most likely choice without user input, and may vary widely in complexity and sophistication. Meta-mediators are used to select the appropriate mediator based on context type, application state, and so on, and help to decide whether mediation should involve the user or not.

A mediator typically handles or displays the same portion of the graph that an application would have acted on had no ambiguity been present. Thus, a mediator may be created by simply extending an existing mediator from our toolkit library to display events that an application was already designed to support, or to resolve ambiguity that would have been resolved before an event reached the application in the past. Mediators all include architectural support for accepting or rejecting events, with the side effect that correct interpretations are kept and incorrect interpretations removed from the graph. Once the ambiguity is resolved, the toolkit allows processing of that portion of the input graph to continue as normal. Typically, a mediator will focus on the highest level interpretations (leaf nodes) of the graph, and accepting a leaf node is sufficient to disambiguate the remainder of the graph because the entire path from the root to that leaf must also be accepted, while any conflicting paths must be rejected [28].

Similarly to OOPS, our toolkit automatically handles the tasks of routing input to mediators when it is ambiguous, and of informing recognizers and the application about the correct result when ambiguity is resolved. This separation of mediation from recognition and from the application means that the basic structure of an application and its interface does not need to be modified in order to add to or change how recognition or mediation is done. Additionally, the directed graph provides a consistent internal model 
that makes it possible to build mediators that are completely independent of recognizers. Note that an application may opt to bypass mediation and receive ambiguous events directly, or ask to only be informed about events that are unambiguous or have had all ambiguity resolved. A non-interactive component that cannot ask the user for input may select this option, for example.

Figure 4 shows the resulting changes. The light gray boxes indicate components that have been added to the Context Toolkit architecture illustrated in Figure 2 to support mediation of ambiguous context.

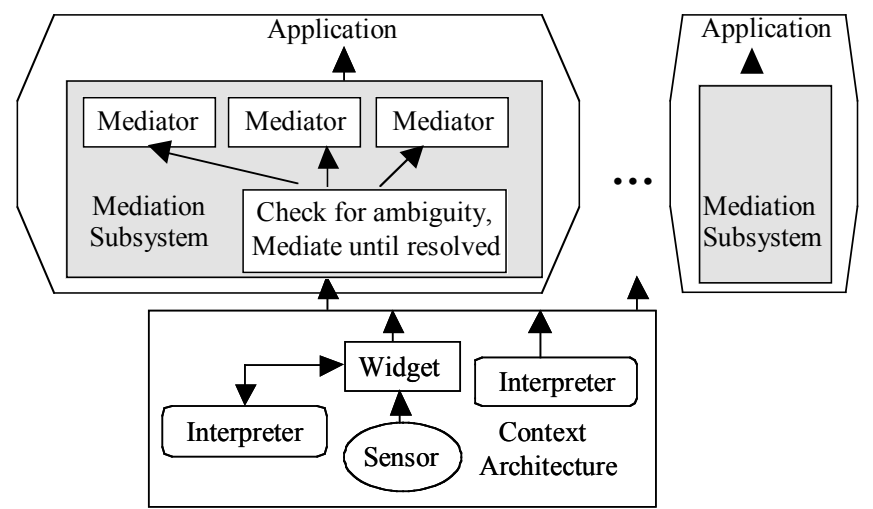

Figure 4 The architecture for the extended Context Toolkit. Everything in the gray boxes is new.

\subsection{Example}

Before discussing the additional changes necessary to support the requirements listed in Section 5, we illustrate the use of ambiguous hierarchical events in the Context Toolkit with an example. In the Communicator system, time and location information is used to choose relevant vocabularies. An intelligent recognition system provides the most likely vocabularies and then these are interpreted into the words the user is most likely to be typing. The set of vocabularies and the set of words are stored as sets of alternatives with associated confidences (a fairly common representation). Each of these alternatives becomes an ambiguous event in our system. The result is a directed graph, like that shown in Figure 3. Eventually, the user will need to select the correct path through this graph (e.g. mall \& Wednesday $\rightarrow$ shopping $\rightarrow$ clothes).

Now suppose that an application subscribes to this data. Subscribers to ambiguous data, using our toolkit, may wait until all the ambiguity has been resolved before taking any action on a location update; or take action on the ambiguous data using an automatic or interactive mediator. In the case of the Communicator, we have chosen to let the user mediate the graphs at two levels: that of vocabularies (which change only when the user changes location), and that of words. A single mediator that displays input events as a row of buttons must be created, inheriting from our basic choice mediator. Two instances of this mediator are instantiated and passed to the architecture. One is assigned to handle 
vocabulary events when they arrive using the same subscription mechanisms that an application would use to select that particular piece of context, while the other is assigned to handle word events when they arrive. Should the user select a vocabulary or word, the event graph will be updated by a call to the system-provided accept ( ) method. When a new set of vocabularies or words is delivered to the application, the architecture automatically routes them to the appropriate mediator, asking the mediator to update its display by replacing the previous set with the new set. When ambiguity is resolved, the main application receives the final choice and may act on it (in this case, by displaying it to the communication partner).

\subsection{Modifications for New Requirements}

The previous subsections described the basic abstractions used to support mediation: widgets, interpreters, applications, mediators and the event graph. These abstractions, taken from our past work [28], were sufficient to handle the first three requirements listed in Section 5 (context acquisition and ambiguity, context mediation, and multiple subscription options). We now explain the additional architectural mechanisms needed to support the remaining three requirements, which all represent unique problems faced by mediation of ambiguous context in a distributed, multi-user setting, introduced above.

4) Pre-emption of Mediation: Because multiple applications may subscribe to the same ambiguous data, mediation of the same data may actually occur simultaneously. If multiple components are mediating at once, the first one to succeed "interrupts" the others and updates them with the mediated data. This is handled automatically by the architecture when the successful mediator accepts or rejects data. The architecture notifies any other recipients about the change in status. The architectural stub in each recipient component that handles communication and mediation determines if the updated data is currently being mediated locally. If so, it informs the relevant mediators that they have been pre-empted and should stop mediating. Our solution is particularly important to supporting mediation over space and time (our second design guideline), since it allows multiple mediation opportunities to be presented simultaneously at different locations along the user's expected path. It is unique architecturally because it handles mediation in multiple distributed components.

5) Forced Mediation: In cases where a subscriber of ambiguous context is unable to or does not want to perform mediation, it can request that another component perform mediation, by passing the set of ambiguous events it wants mediated to a remote component, and have that remote component perform the mediation. If the remote component is unable to do so, it notifies the requesting component. Otherwise, it performs mediation and updates the status of these events, allowing the requesting 
component to take action. Currently, there is no way to request mediation without specifying who should do it. Forced mediation may be used when ambiguity has been retained (the first design guideline), and now needs to be resolved.

6) Feedback: Since context data may be gathered at locations remote from where the active application is executing and at times remote from when the user is interacting with the active application, there is a need for distributed feedback services that are separate from applications. To support distributed feedback, we have extended context widgets to support feedback and actuation via output services. Output services are quite generic and can range from sending a message to a user to rendering some output to a screen to modifying the environment. Some existing output services render messages as speech; send email or text messages to arbitrary display devices; and control appliances such as lights and televisions. Any application or component can request that an output service be executed, allowing any component to provide feedback to a user. This issue is also particularly relevant to supporting mediation over space and time, as well as retaining ambiguity (our second and fourth design guidelines). If and when mediation is necessary, a user may not be in the location where the data was originally sensed, nor where the application using it is located. Remote feedback can help with this.

7) Storage: When context is ambiguous, it is not immediately obvious what should be stored and when. One option is to store all data, regardless of whether it is ambiguous or not. This option provides a history of user mediation and system ambiguity that could be leveraged at some later time to create user models and improve recognizers' abilities to produce interpretations. We chose to implement a less complex option: By default, every widget stores only unambiguous data. As a consequence of this choice, if no applications have subscribed to a sensor, that data it senses will not be stored, and therefore will not be available to applications later. One solution available to a widget designer is to ask the user to disambiguate the data even in the absence of an application. This represents a burden to the user. Another alternative is to automatically choose an interpretation (essentially what is done by default in most toolkits).

Another dimension of storage relates to when data is stored. Since we are storing unambiguous data only, we store context data only after it has been mediated. The reason for our choice is two-fold: the storage policy is easier to deal with from an access standpoint, because applications can treat any retrieved data as certain, and we gain the benefits offered by knowledge of ambiguity during the mediation process without bearing the cost of long-term storage. All that is lost is access to information about 
ambiguity at some arbitrary time after mediation (when the record of ambiguity has been discarded). In any case, it would be relatively simple to modify the architecture to store all information about ambiguity.

\subsection{Summary of Architecture}

\begin{tabular}{|l|l|}
\hline (a) Application & (b) Mediator \\
\hline Specify whether to handle ambiguous data or not & (if interactive) Produce some feedback about the \\
Create subscriptions to widgets & data being mediated \\
Retrieve data from storage, if necessary & $\begin{array}{l}\text { Accept or Reject events (based on user interaction } \\
\text { if interactive) } \\
\text { Install mediators }\end{array}$ \\
Handle results of subscriptions & state if pre-empted or forced to mediate \\
& (c) Widget \\
\hline Specify the data you provide \\
When creating events, specify their sources if appropriate \\
When creating events, if ambiguous, specify each possible interpretation \\
Garbage collect and perform widget-specific actions on mediated data
\end{tabular}

Figure 5 Steps for building system components. Italics represent new steps due to our support for ambiguity. In summary, we have created an architecture that combines the context and distribution capabilities of the Context Toolkit [14] with the ambiguity-handling and mediation capabilities of OOPS [28]. Our combined architecture addresses seven requirements for mediating ambiguous context. The resulting architecture simplifies the previously ad-hoc process of handling ambiguous data, from an application developer's perspective, to the steps shown in Figure 5. Items in italics are additions caused by our support for mediation and ambiguity. Note that Mediators (Figure 5b) and Widgets (Figure 5c) are intended to be re-used and may not need to be created from scratch for every application. This leaves only two, crucial, steps relating to mediation to the typical application developer (Figure 5a): He must decide whether or not the application should receive and handle ambiguous data, or only receive data once mediation has occurred (this requires a single change to the subscription code); and he must select which mediators should be used to resolve ambiguity and install them (typically this requires about two lines of code per mediator).

Mediators are designed to support re-use in multiple applications. However, should the existing mediators in the toolkit library not suffice, an application developer may write a new mediator. This task is separated from application development so that mediators may be easily replaced or modified, allowing application developers to easily experiment with appropriate mediation strategies. However, the basic task of writing a mediator is no more complicated than dealing with ambiguity directly in the application would be, while the benefits in terms of the support for mediation across multiple applications are great. The new mediator can be re-used by other applications and the 
architecture handles the job of communicating the results of the mediation to other interested components. Additionally, the architecture automatically determines when ambiguity is present and mediation is needed, and routes ambiguous events to the appropriate mediator (based on subscriptions created by the application developer).

The designer must find some way to provide feedback to the user about the data being mediated. When the user responds to that feedback, the mediator uses that information to call a single method (accept, or reject), informing the system of the final correct interpretation. Finally, the designer must decide how the mediator should act when preempted or forced to mediate.

As with mediators, a developer may find himself writing a new widget. This task is fairly straightforward even when data is ambiguous. Rather than creating a single event to represent a piece of sensed data, one event is created for each ambiguous interpretation. If any of these events was derived from pre-existing events (this is more common in interpreters than widgets), those source events must be made explicit to the system, by passing them as arguments to the new event's constructor. Finally, when the widget is notified that an event has been mediated, it may wish to perform special actions such as updating a learning algorithm. However, this last step is not required. Note that giving the widgets access to information about ambiguity may allow them to do other interesting things such as fusing data from multiple sensors to create more sophisticated interpretations of user input. However this is not a focus of the current work.

Note that it is straightforward to integrate existing applications that cannot handle ambiguity into this architecture. No mediators need be installed, so the applications themselves only require a change to one constructor. Existing widgets, as described above, must specify the source of any events they create, again by simply changing the arguments to a constructor.

While we have given some details above about how an application programmer would use our architecture, more information on those requirements can be found in our prior work on this topic [13]. In the next section, we describe three applications that we built with this architecture. These applications illustrate the design guidelines presented in Section 4, and those design issues are discussed below.

\section{CASE STUDIES}

In this section, we describe the building of three context-aware applications, the Communicator, an In/Out Board and a reminder system. The first application was built entirely using our new architecture, and includes both ambiguous and unambiguous data sources. The other two were modifications of existing applications to include ambiguous sensors and mediation. These applications validate our architecture and illustrate how our 
design guidelines can be applied to real systems. These applications represent a range of complexity in terms of the number of types of context they use, the mobility of their users and the extent to which sensing is implicit or explicit. Because each application we built represents a range of characteristics typical of existing context-aware applications, we believe our examples are of practical use to designers.

\subsection{The Communicator}

We introduced the Communicator system as our motivating example. Its interface is shown in Figure 1. Here we will describe the physical setup of the application, the interaction supported, the system architecture and how the design guidelines were applied in building this application.

This application demonstrates two important features of the architecture. First, it shows that the architecture supports experimentation with mediation by making it trivial to swap mediators in and out. Adding or replacing a mediator only requires two lines of code. Second, it shows that it is not difficult to build a compelling and realistic application. The main Communicator application consists of only 435 lines of code, the majority dealing with GUI issues. Only 19 lines are for mediation and 30 deal with context acquisition. Additionally, it particularly illustrates the need for appropriate use of defaults, and for retaining ambiguity.

\subsubsection{Physical Setup}

The Communicator runs on a laptop computer, intended to be attached to a wheelchair. A GPS unit is mounted on the wheelchair and connected to the computer. As the user moves from location to location in a downtown city environment, the Communicator suggests appropriate vocabularies based on the current location and time, shown near the bottom of the interface. The user can select a vocabulary at any time during a conversation to help the system predict appropriate words. As the user starts entering characters with the scanning interface, the system predicts potential word completions shown near the top of the interface, using words from the system-suggested vocabularies or the user-selected vocabulary, to support him in maintaining a conversation. The user can select any of the suggested words or continue to type individual characters. If the user is speaking with a companion using a partner device, the companion can also select the appropriate vocabulary on behalf of the user.

\subsubsection{Implementation}


Figure 6 Architecture for the Communicator System

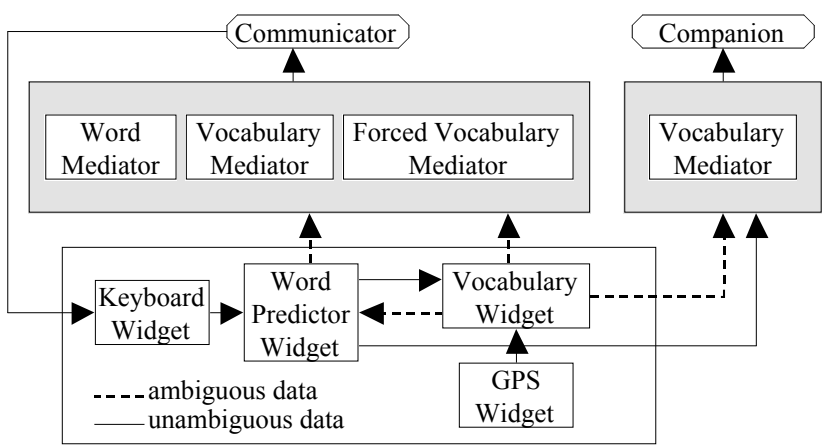

The Communicator makes direct use of data from three widgets: a soft keyboard, a word predictor and a vocabulary selector. The keyboard widget produces unambiguous data and simply lets other components know what the user is typing. The word predictor widget produces ambiguous data and uses the current context to predict what word the user is trying to type. It uses a unigram, frequency-based method common in simple word predictors, as well as a history of recent words. It subscribes to the keyboard to get the current prefix (the letters of the current word that have been typed so far). As each letter is typed, it suggests the most likely completions. The word predictor also uses weighted vocabularies to make its predictions. It subscribes to the vocabulary widget to get a list of ambiguous, probable vocabularies and uses the probability associated with each suggested vocabulary to weight the words from that vocabulary. As described earlier, the vocabulary widget uses the Remembrance Agent [35] to suggest relevant, yet ambiguous vocabularies for the current conversation.

If the person the user is communicating with also has a display available, a companion application can be run. This application presents an interface (see Figure 1), showing the unambiguous words selected by the user and the current set of ambiguous vocabularies.

This application uses two unambiguous widgets (GPS and keyboard), and two widgets that generate ambiguous data, one based on a third party recognizer (vocabulary), and one based on an in-house recognizer (word). Unlike typical context-aware systems, ambiguity in our systems is retained, and, in some cases, displayed to the user.

Ambiguous information generated in our system includes potential vocabularies and potential words. The architecture allows a component to mediate ambiguous context, use it as is, or use it once something else has mediated it. All three cases exist in this system. The application mediates both ambiguous words and vocabularies. The word predictor uses ambiguous vocabularies. The vocabulary widget uses unambiguous words 
after the user has mediated them. The word mediator is graphical and it displays ambiguous words as buttons in a horizontal list, shown in situ near the bottom of Figure 1a. A word may be selected by the user or ignored. The mediator replaces all the displayed words whenever it receives new words from the word predictor.

\subsubsection{Design Issues}

The Communicator supports mediation of both words and vocabularies. For the mediation of words, users can either select one of the words suggested or can continue typing causing a new set of suggested words to appear. We experimented with four different strategies for mediating ambiguous vocabularies. The first simply accepts the vocabulary with the highest probability without user input (equivalent to no mediation at all). The second (see Figure 7a) displays the choices similarly to words, and allows the user to ignore them. The last two require the user to choose a vocabulary at different points in the conversation (Figure $7 \mathrm{~b}$ ). The third requires a choice when a new conversation starts and new ambiguous vocabularies are suggested. The fourth displays the choices, but only requires that the user choose one when a conversation has ended. The mediated vocabulary name is used to append the current conversation to the appropriate vocabulary file, which then improves future vocabulary/word prediction. These approaches demonstrate a range of methods whose appropriateness is dependent on recognizer accuracy. The architecture easily supports this type of experimentation by allowing programmers to easily swap mediators. Providing this range of mediation techniques offers flexibility to the end user.

The variety of mediators we experimented with allowed us to explore two design heuristics: the appropriate use of defaults and retaining ambiguity.

(a)

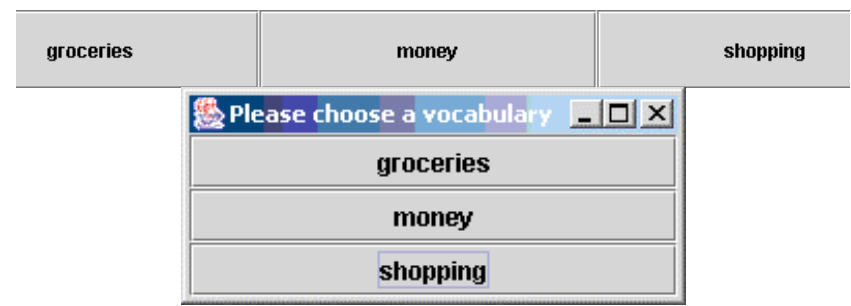

Figure 7 Screenshots of mediators (a) choice mediator for words or vocabularies and (b) required mediator for vocabularies.

Appropriate use of defaults: Providing appropriate defaults and reducing keyboard input is the main purpose of this application. The different mediators we experimented with supported different defaults. The mediator that automatically chose the most likely vocabulary is most appropriate when there is little ambiguity, whereas the mediator that does not require the user to select a vocabulary is most appropriate when there is a 
lot of ambiguity. Because each selection act by the user may take multiple seconds, the appropriate use of defaults is critical in this application.

Retaining Ambiguity: The fourth mediator described above only requires mediation when a conversation ends. This demonstrates how an application can postpone mediation as long as possible. In particular, the conversation needs to be recorded to disk in the correct vocabulary category, so vocabulary ambiguity must be resolved at this time. Again, this guideline supports the goal of minimizing the number of selection requests for the user. Additionally, by allowing the user to postpone mediation as long as possible, we minimize the chance that mediation will interrupt an already slow conversation.

\subsection{In/Out Board}

The In/Out Board [14], an application that displays the current in/out status for occupants of a home. In the original system, an unambiguous location widget informed the application when a user entered or left the building. Users indicated their status by docking a Java iButton ${ }^{\circledR}$. We modified this application to include ambiguity and mediation.

The only direct modification of the original In/Out Board application was to install a mediator and some additional widgets described below. The newly modified widgets used by the In/Out Board are reusable and one of them is, in fact, used by the next application as well. The mediator we use is an extension of a mediator from our library of mediators, modified to display application-specific text. A total of 22 lines were changed or added to the In/Out Board application code. 14 were minor substitutions where references were changed from the unambiguous widget to the ambiguous one and three were new library imports. Two new class variables were created to hold pointers to the mediator and three lines of code were added to create the mediator and pass it one piece of necessary information about the application, a pointer to its user interface. The In/Out Board particularly highlights the use of redundant mediation techniques, spatio-temporal relationship of input and output, and effective use of defaults.

\subsubsection{Physical Setup}

Occupants of the home are detected when they arrive and leave through the front door, and their state on the In/Out Board is updated accordingly. Figure 8(a) shows the front door area of our instrumented room, taken from the living room. In the photographs, we can see a small anteroom with a front door and a coat rack. The anteroom opens up into the living room, where there is a key rack and a small table for holding mail - all typical artifacts near a front door. To this, we have added two ceiling-mounted motion detectors 
(one inside the house and one outside), a display, a microphone, speakers, a keyboard and a dock beside the key rack.

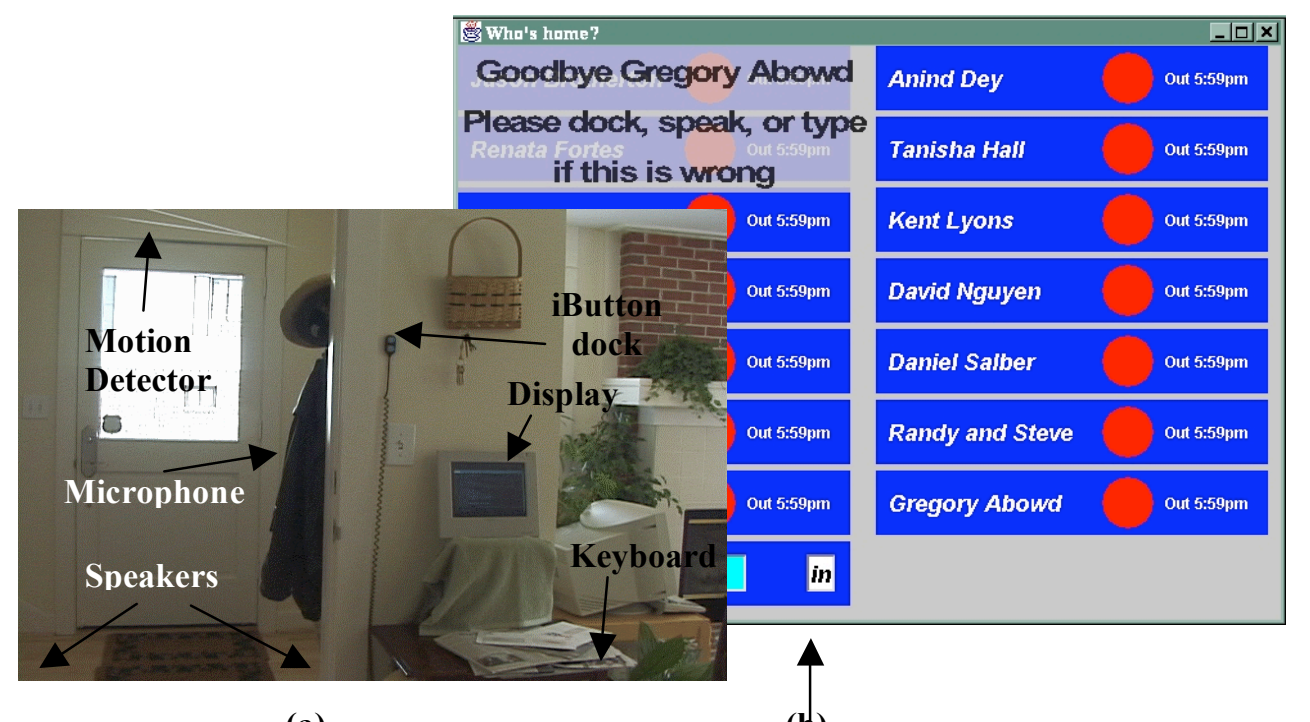

(a)

(b)

Figure 8 (a) Photograph of In/Out Board physical setup; (b) In/Out Board with transparent graphical feedback. 7.2.2 Implementation

The application was originally written using the unmodified Context Toolkit. Figure 9 shows the block diagram of the components in the system. Input captured via context widgets detected presence using an iButton ${ }^{\circledR}$. We modified the application to use additional widgets from the original Context Toolkit: motion detectors, speech recognition, and keyboard widgets. All of the widgets were modified to be able to generate ambiguous as well as unambiguous context information.

Rather than requiring explicit action from the user to determine in/out status, the motion detector-based widget uses an interpreter to interpret motion information into user identity and direction. The interpreter uses historical information collected about occupants of the house, in particular, the times at which each occupant has entered and left the house on each day of the week. This information is combined with the time when the motion detectors were fired and the order in which they were fired. A nearest-neighbor algorithm is then used to infer identity and direction of the occupant. The speech recognition-based widget uses a pre-defined grammar to determine identity and direction.

Because the original system did not support ambiguity, it ignored the fact that docking an iButton ${ }^{\circledR}$ merely provides information about user presence and not about user arrival or departure from a room. The interpreter used by the motion detector widget not only introduces this ambiguity about user state, but, in an attempt to require less explicit user action, also introduces additional ambiguity about the user's identity. The motion 
detector widget makes this information available to the rest of the architecture and application.

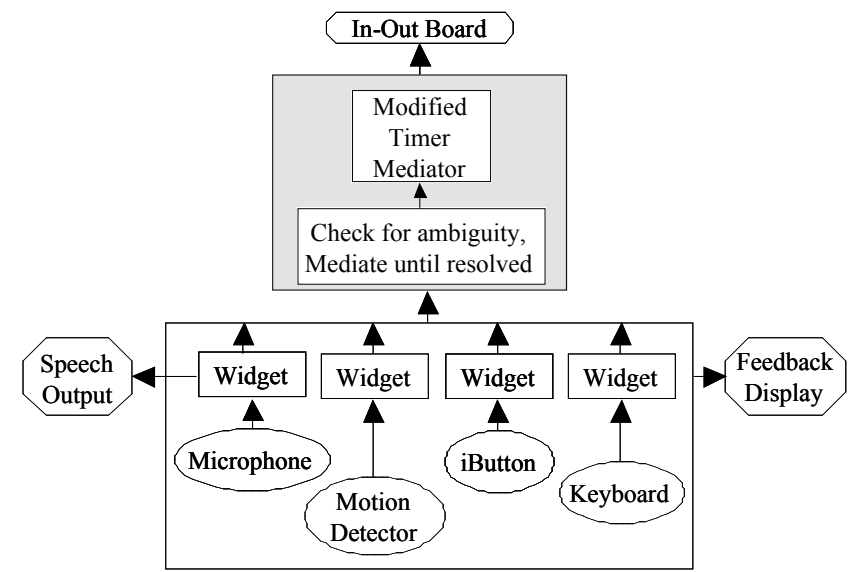

Figure 9 Architecture diagram for In/Out Board. There are 4 widgets providing context, two of which have output services for feedback: speech output and visual feedback display.

The mediator we designed displays the current best guess to the user as she enters the foyer (Figure 8b), and allows her to correct it in a variety of modalities ranging from lightweight to heavyweight, including speech, docking her iButton ${ }^{\circledR}$, and typing at a keyboard. First, a mediator speaks the most likely inference using synthesized speech, using the feedback service provided by our architecture. For example, it might say "Hello Jane Doe" or "Goodbye Fred Smith". In addition, the wall display shows a transparent graphical overlay (see Figure 8(b)) indicating the current state and how the user can correct it if it is wrong: speak, dock or type.

If the inference is correct, the individual can simply continue on as usual. After a timeout period of 20 seconds, the mediator will select the top choice and the In/Out Board will be informed and update its display with this new information. If the inference is incorrect, the direction (exiting or entering) and/or identity may be wrong. The individual can correct the inference using a combination of speech input, docking with an iButton, and keyboard input on the display. For example, the user can say things like "No", “No, I'm entering/exiting”, “No, it's John Doe”, or "No, it's John Doe and I'm entering/exiting". These input techniques plus the motion detectors range from being completely implicit to extremely explicit. Each of these techniques can be used either alone, or in concert with one of the other techniques. There is no pre-defined order to their use. Since some of these inputs may themselves be ambiguous, the mediator responds to the refinements by resetting a timer, and providing additional feedback indicating how the new information is assimilated, by speaking and displaying the change. 
Changes can continue to be made indefinitely, however, if the user makes no change for a pre-defined amount of time, mediation is considered to be complete. As the timer counts down, the transparent display slowly fades away. When the timer expires, the current choice is selected and the service updates the wall display with the corrected input. The timeout for this interaction is 20 seconds.

The mediator is an instance of a temporal mediator from our toolkit library, which creates a timer to create temporal boundaries on this interaction. The timer is automatically reset if additional input is sensed before it runs out. As the mediator collects input from the user and updates the graph to reflect the most likely alternative, it provides feedback to the user.

\subsubsection{Design Issues}

In this section, we will further investigate the design guidelines that arose during the development of this service.

Redundant mediation techniques: On the input side, we attempted to provide a smooth transition from implicit to explicit input techniques. Often users will have their hands full, so speech recognition is added as a form of more explicit, hands-free input. IButton docking provides an explicit input mechanism that is useful if the environment is noisy. Finally, keyboard input is provided as an additional explicit mechanism and to support the on-the-fly addition of new occupants and visitors. While we were initially tempted to simply build a single, best, mediator, we believe the best solution was a combination of mediation techniques, because it greatly increases the level of flexibility available to the user. Our use of the system showed that redundancy also helped when mediation failed due to additional ambiguity. For example, ambient noise, or recognition errors, often caused the speech mediator to fail.

Spatio-temporal relationship of input and output: The next design decision is where to place the input sensors and the rendering of the output to address the spatio-temporal characteristics of the physical space being used. There are "natural" interaction places in this space, where the user is likely to pause: the door, the coat rack, the key rack and the mail table. The input sensors were placed in these locations: motion sensors on the door, microphone in the coat rack, iButton dock beside the key rack and keyboard in a drawer in the mail table. The microphone being used is not high quality and requires the user to be quite close to the microphone when speaking. Therefore the microphone is placed in the coat rack where the user is likely to be leaning into when hanging up their coat. A user's iButton is carried on the user's key chain, so the dock is placed next to the key rack. The speakers for output are placed between the two interaction areas to allow it to be heard throughout the interaction space. The display is placed 
above the mail table so it will be visible to individuals in the living room and provide visual feedback to occupants using the iButton dock and keyboard. If we had put all of the sensors in one location, the user would have been forced to stay in that location in order to complete mediation, interrupting her other tasks.

Effective use of defaults: Another design issue is what defaults to provide to minimize required user effort. We use initial feedback to indicate to the user that there is ambiguity in the interpreted input. Then, we leave it up to the user to decide whether to mediate or not. The default is set to the most likely interpretation, as returned by the interpreter. Through the use of the timeout, the user is not forced to confirm correct input and can carry out his normal activities without interruption. This is to support the idea that the least effort should be expended for the most likely action. The length of the timeout, 20 seconds, was chosen to allow enough time for a user to move through the interaction space, while being short enough to minimize between-user interactions. By using effective defaults, we minimize the burden on the user who would otherwise have to confirm her presence each time she entered or left the house, even though the system provides her no useful services at that moment.

Other design issues: We added the ability to deal with ambiguous context, in an attempt to make these types of applications more realistic. Part of addressing this realism is dealing with situations that may not occur in a prototype research environment, but do occur in the real world. An example of this situation is the existence of visitors, or people not known to the service. To deal with visitors, we assume that they are friendly to the system, a safe assumption in the home setting. That means they are willing to perform minimal activity to help keep the system in a valid state. When a visitor enters the home, the service provides feedback (obviously incorrect) about who it thinks this person is. The visitor can either just say "No" to remove all possible alternatives from the ambiguity graph and cause no change to the display, or can type in her name and state using the keyboard and add herself to the display.

\subsection{CybreMinder}

The last application we created is CybreMinder [15], a situation-aware reminder system. The original application subscribes to every widget that is running in the Context Toolkit, using a discovery system, and allows the user to create reminders for themselves or someone else triggered by any situation, or combination of events, that these widgets might generate. For example, a user might set up a reminder to go to a meeting when at least three other people are present in the meeting room at the right time. Delivery of reminders is performed whenever the current context appears to match the triggers 
specified by the user. The application assumes that the reminder has been successfully delivered and acted upon. The purpose of this system is to trigger and deliver reminders at more appropriate opportunities than is currently possible.

Users can create situation-aware reminders on any networked device. For example, suppose Jane and David are working on a paper. Jane sends out a draft of the paper and is waiting for comments. She creates a reminder message for David to drop off his comments. She sets the situation in which to deliver the reminder to be when David enters the building.

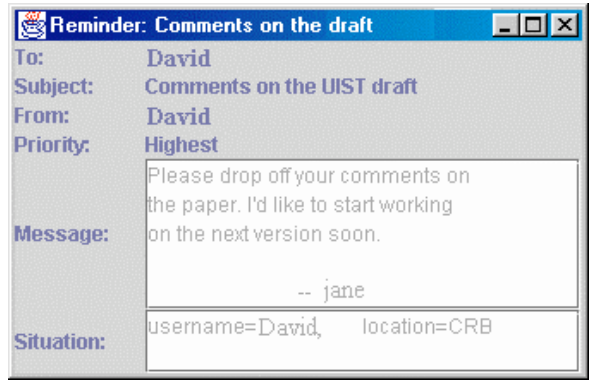

Figure 10 Reminder message delivered in appropriate situation.

When David enters the building (sensed by the appropriate iButton dock), the reminder is delivered to him on the closest display that also beeps to get his attention (Figure 10). Just because the reminder was delivered to David, does not mean that he will complete the action detailed in the reminder. In fact, the most likely occurrence in this setting is that a reminder will be put off until a later time. The default status of this reminder is set to "still pending". This means that the reminder will be delivered again, the next time David enters the building. However, if David does enter Jane's office within a pre-defined amount of time (10 minutes), as indicated by him docking his iButton, the system changes the previous incorrect reminder status to "completed" and the application is informed that the reminder has been delivered (Figure 11a). Of course, if he was just stopping by to say hello, he can dock again to return this back to "still pending" (Figure 11b).

(a)

The status of your reminder 'Comments on the draft' is now: delivered
Theminder Status Update

(b) Reminder Status Update The status of your reminder 'Comments on the draft' is now: pending

Figure 11 Graphical reminder status: (a) "delivered" and (b) "pending".

The original CybreMinder application was only modified to subscribe to all iButton ${ }^{\circledR}$ widgets so the application would be notified when a user docked to mediate a reminder and to install a reusable temporal mediator that selects and updates the most likely interpretation after a timeout (as in the case of the $\mathrm{In} /$ Out Board). The mediator makes use of remote widget feedback services to display feedback about the reminder 
status. It is an extension of a timer mediator modified to display application-specific messages. The application modifications required the addition of 3 library imports and the modification or addition of 27 lines of code. This application particularly highlights the effective use of defaults, spatio-temporal relationship of input and output and redundant mediation techniques.

\subsubsection{Physical Setup}

Various locations (building entrances and offices) in two buildings have been instrumented with iButton docks to determine the location of building occupants. With each dock is a computer screen on which reminder messages can be displayed. Figure 12 shows an example installation.

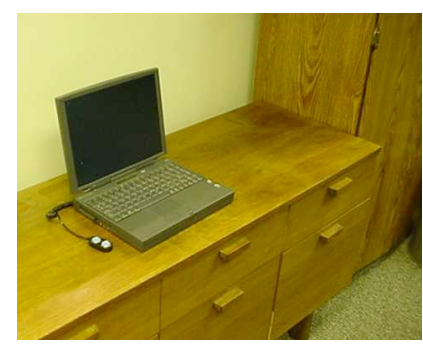

Figure 12 Reminder display with iButton dock.

\subsubsection{Implementation}

Figure 13 shows the block diagram of the components in this system. Input is captured via context widgets from the toolkit library that detect presence using iButtons as the input-sensing mechanism. When the information from these widgets matches a situation for which there is a reminder, the reminder is delivered to a display closest to the recipient's location. The reminder is displayed using a remote feedback service that the widgets provide. Initially, input in this system was treated as unambiguous in [15], however in our updated architecture, the iButton widget creates a pair of ambiguous events, one indicating that the reminder is still pending and one indicating that the reminder has been completed.

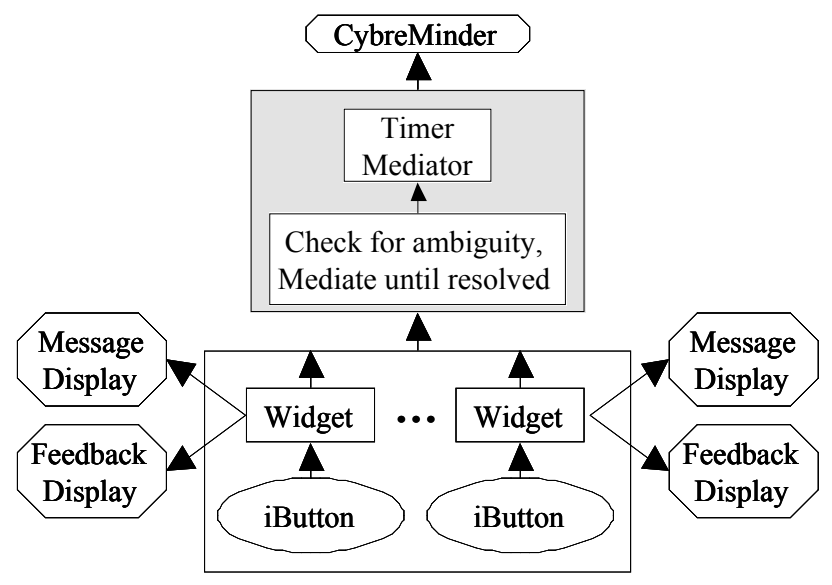


Figure 13 Architecture diagram for reminder service. There is one widget for each "interesting" location in the building. Each widget has two services, one for displaying a reminder and one for providing feedback about the reminder's status.

When ambiguous events arrive, they are delivered to a mediator that gives the user the opportunity to accept a reminder by docking his iButton within a certain time after its delivery, in which case the system proceeds to change its status to 'delivered' just as it would have done immediately in the original application. The mediator uses a timer to enforce temporal boundaries of 10 minutes on the mediation process. The mediator attempts to get the user's attention via an audio cue and on the display that is closest to the user's current location (Figure 12) using a feedback service provided by the local widget.

\subsubsection{Design Issues}

When designing this service, we chose to address the ambiguity only at the level of whether the reminder was dealt with or not. This was done in order to make the design simpler for demonstration purposes. The underlying context that is used to determine when a message should be delivered will also have ambiguous alternatives that may need mediation. It should not be hard to see how we could combine the type of service we demonstrated with the In/Out Board with the reminder service, to make this possible. In terms of our existing guidelines, the following decisions were made:

Redundant mediation techniques: We use redundant output techniques, including a simple audio cue to get the user's attention, and a visual cue that indicates the current reminder status. A visual cue alone does not suffice because the user may not see it. The additional sound cue notifies the user that there is something to pay attention to and the visual cue provides the necessary content. Although this may be an issue in desktop settings, it is exacerbated when the user is mobile. Speech input was not used in this setting because it was a more public space than the home. In addition to the docking-based mediation described above, a user can explicitly correct the status of a reminder using a separate interface from the comfort of her desk.

Spatio-temporal relationship of input and output: The choice of locations for input and output was again guided by the space in which the service was deployed. Natural locations for sensing input and providing feedback were the entrances to rooms where users would naturally stop to dock anyway. If a different location system were used, the locations chosen might differ slightly. Entrances to offices would still be appropriate, as they are natural stopping places where users knock on a door. But in a conference room, the chairs where users sit may be a better choice. Rather than expecting the user to remain in a single location for mediation, this guideline led us to identify key locations for bringing the interaction to the user. 
Effective use of defaults: In this service, as opposed to the In/Out Board, the default interpretation of user input is that no action is taken and the reminder is still pending. This default is chosen because the implicit user input received (a user entering the building) only causes a reminder to be delivered, and does not provide any indication as to whether the reminder has been addressed and completed. There is really no sensor or group of sensors that will enable us to accurately determine when a reminder has been addressed. We must rely on our users to indicate this. The timeout for accepting the input was chosen to be long enough to give the user an opportunity to address the reminder, while short enough to minimize overlap between individual interactions (reminders).

Retaining ambiguity: The CybreMinder application does not try to resolve any ambiguity except whether the reminder has been delivered and acted upon. If other ambiguity exists, it does not request any mediation. Rather, it waits until that ambiguity has been resolved by other applications or components. An alternative would be to simply display all potential reminders rather than mediating the question of what reminder to show. Because the application subscribes to every available sensor, mediating every piece of ambiguous data would be an impossible burden for the user.

\subsection{In Summary}

We built one new application and modified two existing applications. These applications represent a range of complexity within the context-aware domain. Between them, they demonstrate all the required features of the architecture. They use (and reuse) a number of ambiguity-generating widgets. The first application was new and very little of its code was dedicated to dealing with mediation or context acquisition. The other two applications required minor modifications to deal with ambiguity. Mediation in all three cases was designed with our guidelines in mind. The applications help to illustrate the validity of the underlying architecture and to demonstrate how the design guidelines can be analyzed and applied in real settings.

\section{FUTURE WORK}

The extended Context Toolkit supports the building of more realistic context-aware services that are able to make use of ambiguous context. But, we have not yet addressed all the issues raised by this problem.

Because multiple components may subscribe to the same ambiguous events, mediation may actually occur simultaneously in these components. When one component successfully mediates the events, the other components need to be notified. We have already added the ability for input handlers to keep track of what is being mediated locally in order to inform mediators when they have been pre-empted. Although 
we have implemented this basic algorithm for handling multiple applications attempting to mediate simultaneously, we would like to add a more sophisticated priority system that will allow mediators to have control over the global mediation process. This could also support more sophisticated ways of dealing with conflicts when multiple applications or users are mediating the same data.

Related to this issue of multiple applications mediating, is the need to examine whether a single, final mediated result is appropriate for multiple applications. An alternative would be to modify our architecture to maintain information about multiple disambiguations for different applications or groups of applications. There may be situations where a user may want different mediated results to be sent to different applications, to protect her privacy, for example. In an application located within her home, she may be willing to provide exact information about her activities, but may wish to provide less fine-grained or even false information to an application to applications and users outside her home. By building more applications that use mediated information, we hope to understand this issue more fully.

An additional issue we need to further explore is how events from different interactions can be separated and handled. For example, in the In/Out Board service, it is assumed that only one user is mediating their occupancy status at any one time. If two people enter together, we need to determine which input event belongs to which user in order to keep the mediation processes separate.

We also plan to build more context-aware services using this new architecture and put them into extended use. This will lead to both a better understanding of how users deal with having to mediate their implicit input and a better understanding of the design guidelines involved in building these context-aware services.

\section{CONCLUSIONS}

The extended Context Toolkit supports the building of realistic context-aware services, ones that deal with ambiguous context and allow users to mediate that context. When users are mobile in an aware environment, mediation is distributed over both space and time. As a result of this, the design of mediation differs from the GUI domain. We introduce design guidelines for mediating distributed, context-aware applications.

- Applications should provide redundant mediation techniques to support more natural and smooth interactions;

- Applications should provide facilities for providing input and output that are distributed both in space and time to support input and feedback for mobile users; 
- Interpretations of ambiguous context should have carefully chosen defaults to minimize user mediation, particularly when users are not directly interacting with a system.

- Ambiguity should be retained until mediation is necessary for an application to proceed.

To support the mediation of ambiguous context, we extended the Context Toolkit to support seven key features:

- Acquisition of ambiguous context;

- Context mediation;

- Delivery of ambiguous context to multiple applications that may or may not be able to support mediation;

- $\quad$ Pre-emption of mediation by another application or component;

- Applications or services in requesting that another application or service mediate;

- Distributed feedback about ambiguity to users in an aware environment; and,

- Delayed storage of context once ambiguity is resolved.

We demonstrated and evaluated the use of the extended toolkit by modifying two example context-aware applications and by creating a new context-aware application. We showed that our architecture made it relatively simple to create more realistic contextaware applications that can handle ambiguous context and demonstrated the use of the design guidelines for creating these types of applications.

\section{ACKNOWLEDGMENTS}

We would like to thank our colleagues at Georgia Tech and UC-Berkeley who helped us build the applications described in this paper, who used the applications and who provided guidance on the design of the architecture. This material is based upon work supported by the National Science Foundation under Grant No. IIS-0205644.

\section{REFERENCES}

1. ABOWD, G.D. 1999. Classroom 2000: An experiment with the instrumentation of a living educational environment. IBM Systems Journal 38, 4, 508-530.

2. ABOWD, G.D. et al. 1997. Cyberguide: A mobile context-aware tour guide. Balzer/ACM Wireless Networks 3, 5, 421-433.

3. BELLOTTI, V. et al. 2002. Making sense of sensing systems: Five questions for designers and researchers. In Proceedings of CHI 2002, 415-422.

4. BOBICK, A. et al. 1999. The KidsRoom: A perceptually-based interactive and immersive story environment. PRESENCE 8, 4, 367-391.

5. BROWN, M. 1996. Supporting user mobility. In Proceedings of IFIP World Conference on Mobile Communications, 69-77.

6. BROWN, P.J. 1996. The stick-e document: A framework for creating context-aware applications. In Proceedings of Electronic Publishing '96, 259-272.

7. BROWN, P.J., BOVEY, J.D. AND CHEN, X. Context-aware applications: From the laboratory to the marketplace. IEEE Personal Communications 4, $5,58-64$. 
8. CHEVERST, K. et al. 2000. Developing a context-aware electronic tourist guide: Some issues and experiences. In Proceedings of CHI 2000, 17-24.

9. CLARK, H. AND BRENNAN, S.E. 1991. Grounding in communication. Perspectives on socially shared cognition. Resnick, L., Levine, J. and Teasley, S., Ed. American Psychological Society. 127-149.

10. COHEN, P.R. et al. 1997. QuickSet: Multimodal interaction for distributed applications. In Proceedings Of Multimedia '97, 31-40.

11. COOPERSTOCK, J., FELS, S., BUXTON, W. AND SMITH, K. 1997. Reactive environments: Throwing away your keyboard and mouse. $C A C M$ 40,9, 65-73.

12. DAVIES, N., et al. 1997. Limbo: A tuple space based platform for adaptive mobile applications. In Proceedings of Conference on Open Distributed Processing /Distributed Platforms '97.

13. DEY, A.K., et al. 2002. Distributed mediation of ambiguous context in aware environments. In Proceedings of UIST 2002, 121-130.

14. DEY, A.K., SALBER, D. AND ABOWD, G.D. 2001. A conceptual framework and a toolkit for supporting the rapid prototyping of contextaware applications. Human-Computer Interaction Journal 16, 24, 97-166.

15. DEY, A.K. AND ABOWD, G.D. 2000. CybreMinder: A context-aware system for supporting reminders. In Proceedings of the International Symposium on Handheld and Ubiquitous Computing, 172-186.

16. DEY, A.K. AND ABOWD, G.D. 2000. Towards a better understanding of context and context-awareness. In CHI 2000 Workshop on the What, Who, Where, When, Why and How of Context-Awareness.

17. DEY, A.K., ABOWD, G.D. AND WOOD, A. 1999. CyberDesk: A framework for providing self-integrating context-aware services. Knowledge-Based Systems 11, 3-13.

18. ELROD, S. et al. 1993. Responsive office environments. CACM 36, 7, 84-85.

19. FERGUSON, G. AND ALLEN, J.F. 1998. TRIPS: An intelligent integrated problem-solving assistant. In Proceedings of the Fifteenth National Conference on Artificial Intelligence (AAAI-98), 567-573.

20. HARTER, A., et al. 1999. The anatomy of a context-aware application. In Proceedings of Mobicom '99, 59-68.

21. HEER, J. et al. 2003. Presiding over accidents: System mediation of human action. In Proceedings of CHI 2004, CHI Letters. 5, 1, To Appear.

22. HORVITZ, E., et al. 2003. Models of attention in computing and communications: From principals to applications. Communications of the ACM 46, 3, 52-59.

23. HORVITZ, E. 1999. Principles of mixed-initiative interaction. In Proceedings of CHI'99, 159-166.

24. HULL, R., NEAVES, P. AND BEDFORD-ROBERTS, J. 1997. Towards situated computing. In Proceedings of International Symposium on Wearable Computers, 146-153.

25. KORTUEM. G., SEGALL, Z. AND BAUER, M. 1998. Context-aware, adaptive wearable computers as remote interfaces to 'intelligent' environments. In Proceedings of the International Symposium on Wearable Computers, 58-65.

26. LESHER, G.W., MOULTON, B.J. AND HigGinbOThAM, J. 1998. Techniques for augmenting scanning communication. Augmentative and Alternative Communication 14, 81-101.

27. PAEK, T. AND HORVITZ, E. 2000. Conversation as action under uncertainty. Proceedings of UAI-2000, 455-464.

28. MANKOFF, J., ABOWD, G.D. AND HUDSON, S.E. 2000. OOPS: A Toolkit Supporting Mediation Techniques for Resolving Ambiguity in Recognition-Based Interfaces. Computers and Graphics 24, 6, 819-834.

29. MCKINLAY, A., et al. 1995. Augmentative and alternative communication The role of broadband telecommunications. IEEE Transactions on Rehabilitation Engineering 3, 3, 254-260.

30. MORAN, T.P and DOURISH, P. 2001. eds. Special Issue on Context-Aware Computing. Human-Computer Interaction Journal 16, 2-4, 87-420.

31. MOZER, M. C. 1998. The neural network house: An environment that adapts to its inhabitants. In Proceedings of the AAAI Spring Symposium on Intelligent Environments, 110-114.

32. MYERS, B.A. AND KOSBIE, D.S. 1997. Reusable hierarchical command objects. In Proceedings of CHI '97, 260-267. 
33. PASCOE, J., RYAN, N.S. AND MORSE, D.R. 1998. Human-ComputerGiraffe Interaction - HCI in the Field. In Proceedings of the Workshop on Human Computer Interaction with Mobile Devices.

34. REKIMOTO, J., AYATSUKA, Y. AND HAYASHI, K. 1998. Augmentable reality: Situated communication through physical and digital spaces. In Proceedings of the International Symposium on Wearable Computers. 68-75.

35. RHODES, B. 1997. The Wearable Remembrance Agent: A system for augmented memory. Personal Technologies 1, 1, 218-224.

36. SAUND, E. AND LANK, E. 2003. Stylus input and editing without prior selection of mode. Proceedings of UIST 2003, CHI Letters. 5, 2, 213-216.

37. SCHILIT, W.N., 1995. System architecture for context-aware mobile computing, Ph.D. Thesis, Columbia University, May 1995.

38. WANT, R. et al. 1992. The Active Badge location system. ACM Transactions on Information Systems 10, 1, 91-102.

39. WEISER, M. 1991. The computer for the 21st century. Scientific American $265,3,66-75$. 